as an interdisciplinary subject in a modern context. For many years, there has been a clear need for a teaching textbook with a non-specialist approach and with an emphasis on modern concepts, and this work is aimed at this gap. The authors maintain that the development of electrochemistry has been seriously retarded by an overemphasis on the classical thermodynamic approach, and they seek, with crusading zeal, to correct this. As a result, a number of old battles are fought and the sound of this strife may well puzzle younger ears. The importance and utility of the Nernstian heritage are purposely neglected, and at times derided, and, in this sense, the book is unbalanced. The positive features of the work, however, are many and must be stressed.

The first volume gives a lucid and lively description of ionic solutions and it includes a large section on molten salts and glasses. The reader is led gently and expertly through a long series of interlocking discussions on the structure of water, ion-solvent interactions and ion-ion interactions in dilute and in concentrated solutions. The approach emphasizes modern, mechanistic descriptions, it is constructively critical and deals very effectively with the quantitative aspects. The material is built up gradually from an elementary level with a certain amount of repetition, to meet the needs of beginners and of those from outside the field who are seeking electrochemical expertise relevant to their own discipline; thus the book is long, but it is not tedious. Each chapter is divided into short, headed and indexed sections, and good lists of further reading material are provided at appropriate intervals.

The second volume deals in a similar manner with electrified interfaces and electrode processes. The content is generally excellent; for example, it includes a treatment of the semiconductor-electrolyte interface and of electrokinetic properties, and a discussion of electrocatalysis, electrodeposition, corrosion, fuel cells and batteries, but it is surprising in a modern text that no space is found for organic electrochemistry, an important and rapidly growing subject in which many practitioners are nonelectrochemists in need of guidance. Much of the material lies close to the research interests of the authors, and, inevitably, their particular viewpoint on a number of topies is apparent. Their preference occasionally becomes obtrusive in the selection of scientists to be described as the most important contributors to the development of a particular branch of the subject.

The racy, colloquial style is easy to read and is seldom clumsy or obscure. The authors' predilection for coining now names is usually harmless, but there is no justification for neglecting the established terms cathode, anode, reduction and oxidation and substituting electron source electrode, electron sink electrode, electronation reaction and deelectronation reaction. The scope for electrochemistry and its importance in our technologically developing society is discussed at points throughout the books in a stimulating manner, although some of the examples used are very speculative. I am sure this work will substantially aid the vigour and lucidity of the teaching of electrochemistry at the undergraduate and postgraduate levels, and it will be welcomed by many research workers from other fields.

A. BEwICK

\section{BASIC QUANTUM MECHANICS}

\section{Quantum Mechanics}

An Introduction. By J. G. Taylor. (Unwin Studies in Physics.) Pp. 207. (Allen and Unwin: London, June 1970.) $50 s$ boards; $25 s$ paper.

Tars book is meant to cover a one year course for students who are meeting quantum mochanics for the first time and who will not necessarily specialize in theoretical physics. This sensible aim is roughly that of the existing books by Matthews, Strauss and Ziock, for example. Taylor's book stands the comparison well. To begin with, its price is right: you get a lot of quantum mechanics for the price of the paperback edition. For the most part, it is a businesslike presentation of basic quantum mechanies. The book begins, very wisely these days, with a concise summary of classical mechanics. The Schrödinger equation and interpretative postulates are then introduced, and the standard one- and three-dimensional potential problems are mentioned. Time-independent and (an omission in Matthews) time-dependent perturbation theory is discussed, as in Strauss, in the context of atoms and molecules. The sixth chapter contains a much fuller account of scattering theory than either Matthews or Ziock offer, using the Green function method, and the book concludes with a pointer towards the Dirac equation and field theory.

To me the book seems to be particularly suitable for the student who is, in fact, going to specialize in theoretical physics; for such, the two or three page hints about variational principles, linear self-adjoint operators on the Hilbert space $L_{2}\left(\boldsymbol{R}^{3}\right)$, the rotation group and the analytically continued S-matrix would make sense. Although there are some useful analytical problems at the end of each chapter, there are very few numerical ones (as compared with Ziock or Strauss); also one might have reservations on whether the very brief discussion of non-relativistic spin formalism is adequate, especially when compared with Ziock's treatment. But Taylor's book, good value for money, is well worth considering.

I. J. R. AITCHISON

\section{ABSORBED ENERGY}

\section{Photophysics of Aromatic Molecules}

By John B. Birks. (Wiley Monographs in Chemical Physics.) Pp. xiii + 704. (Wiley (Interscience): London and New York, May 1970.) 210 s.

DR BrRKs is a well known experimental physicist in the field of phosphorescence and fluorescence in liquids and solids and he is especially interested in the relative efficiencies and rates at which these processes occur. Photophysics as opposed to photochemistry is not a word I have met previously. The author explains it as being concerned with the physical effects of non-ionizing radiation, but he has restricted himself largely to the subsequent history of the absorbed energy. He does not cover in any detail allied physical properties such as zero-field splitting and the electron resonance of triplet states, the absorption spectra of excited molecules, flash photolysis, and the like. Not that these subjects are totally ignored, but they are only mentioned where they relate to the principal themes of fluorescence, radiationless decay, internal conversion and phosphorescence. Photoconductivity, for instance, is covered in less than two pages.

"Aromatic Molecules" in the title also has a narrow meaning, because heterocyclic molecules, and therewith all $\sigma$ lone pair excitations, are largely excluded. But in the selected field of benzene and the condensed aromatic hydrocarbons such as anthracene and their substituted derivatives the coverage is thorough. Much information is given in tabular form with consistent units and nomenclature, while the text comments helpfully on superseded work, and cases where impurities are suspected or imperfect techniques were used. Anybody requiring up to date numerical values of fluorescence or phosphorescence yields or life times, quenching constants, spectral peak energies or similar information for any carbocyclic aromatic molecule, its excimers, its donor-acceptor complexes in either crystal, glass or solution states is likely to find a reliable number here.

All this is excellent for those with closely related 